\title{
The Role of Logic in Philosophy of Science
}

\author{
Diderik Batens \\ Centre for Logic and Philosophy of Science \\ Ghent University, Belgium \\ Diderik.Batens@UGent.be
}

March 8, 2006

\section{Introduction}

For Logical Empiricism logic was the clue to separating sound reasoning from unsound reasoning and this separation was fundamental, first for understanding science, and next for demarcating science. So logic, formal logic that is, was central for the philosophy of science. The situation changed with the advent of the historicist movement. Science was seen by this movement as contentdriven, as contextual. The role of formal logic was reduced to checking deductive inferences. Logic had nothing interesting to contribute to the mechanisms that are responsible for scientific change.

Shapere (2004) offers an interesting analysis of the reasons why both movements were bound to fail, of the roots of the difficulties, and of their solution. A crucial statement is that "the content of the science that is accepted at any given epoch provides the reasons guiding, and sometimes driving, further inquiry" (p. 50). So science is content-guided: the basis for scientific reasoning is "what we have learned, including what we have learnt about how to learn" (p. 52). By viewing science thus, philosophers of science will avoid to see the scientific method, or the possible scientific methods, as identifiable a priori and, at the same time, will escape the relativism of the historicist movement. This view has been gaining wide adherence during the last decennia.

One might conclude that this view still heavily restricts the role of logic, viz. to avoiding mistaken deductive inferences, but I shall try to show that this conclusion is mistaken. Precisely because science is content-guided, articulating a precise philosophy of science requires a heavy import of logic. It moreover requires intense creative work in logic.

In the sequel of the paper, I shall mainly deal with methodological issues. Before getting there, however, it is useful to briefly discuss the issue of the standard logic.

\section{The Standard Logic}

We need logic for avoiding mistaken deductive inferences, but which logic? First order classical logic (henceforth CL) is clearly best established and most widely promoted by logicians. However, many logicians do not accept CL as "the 
true logic." Intuitionists and (mathematical) constructivists see intuitionistic logic as the standard in mathematics, and sometimes as the general standard. Relevant logicians have argued that CL is mistaken in several respects and that the true logic is a relevant one. Dialetheists argue that there are true contradictions and hence that the true logic should be paraconsistent, viz. not validate the inference from a contradiction to arbitrary statements (from $A$ and not- $A$ to derive $B$ ). And a number of logics, actually too many to even mention the most representative ones, have been presented for specific purposes. The sciences hardly played any role in most of these proposals - quantum logic is an exception. The driving arguments came from insights in everyday language, from metaphysics, and from the history of logic.

So the logic community is not much help in pointing out the true logic. But is there a true logic? Logic determines the meaning of a set of 'logical words' such as 'and', 'not', 'for all', etc. These words are part of languages by which humans try to get a grasp on the world. But choosing a language does not warrant that it is suitable for correctly describing the world. The transition from Newtonand-Maxwell to relativity requires that the language is modified ("conceptual change"). The same holds apparently for every scientific revolution and even for less drastic scientific changes. The point is hardly contentious; decennia ago Hempel acknowledged (in "The theoretician's dilemma") that conceptual change is just as legitimate a move as a replacement of accepted statements within the current conceptual system. If the meaning of logical words does not form an exception in this respect, then only the future history of science can determine which is the true logic.

Every logic contains certain presuppositions about the world. Thus CL presupposes, among many other things, that the world is consistent (that no $A$ is true together with not- $A$ ). Remark, however, that scientific reasoning should enable us to derive conclusions from, among other things, statements that we have reasons to accept on the basis of our best scientific insights. Clearly, stipulating that inconsistencies are false cannot exclude that the available data together with the accepted theories might provide reasons to accept $A$ as well as reasons to accept not- $A$. Moreover, there are historical cases, both from mathematics and from the empirical sciences, in which reasons to accept a statement as well as its negation were present - for references to case studies see (Meheus 2002).

Some will argue that, if we have a reason to accept $A$ as well as a reason to accept not- $A$, then at least one of the reasons is bound to be a bad one. This means that, if a scientific discipline is in an inconsistent state, then one should try to reform it and bring it to a consistent state. I largely agree to this (Batens 2002). It is crucial, however, that, in order to transform the inconsistent state to a consistent state, one needs to reason from the inconsistent state, were it only in order to locate the inconsistencies. To do so in terms of CL is impossible.

Analogous arguments apply to the other presuppositions of CL and, more generally, to the presuppositions of every logic L. The world may resist being grasped by a language of which the logical words are governed by L. In this sense, the true logic is at best the logic that would underlie a complete and correct science, and hence cannot be known at this moment. Meanwhile, however, even if we were to know this true logic, it would be of little use to us, because we have to reason from present-day science in order to improve it. 


\section{Methodological Concepts}

Philosophers of science want to define their concepts in a precise way. Logic is often a good means for doing so. By phrasing the definition in terms of a formalized language, the definition becomes more transparent. Next, the metatheory of logic provides a set of clear tools: the consequence relation, logical relations between statements, model, contradiction, and so on. Where required, logic is extended with set theory, probability theory, and similar mathematical structures. Many nice illustrations of all this are found in Kuipers (2000). Remark that logic does not only enable one to attain a high level of precision. It moreover functions as a heuristic tool: it suggests ways of looking at the problems and of categorizing them; it provides possible relations between statements, sets of statements, and the like; it facilitates seeing the consequences of proposed solutions, ... The most technically elaborated proposal of the sort considered is probably the belief-revision approach as applied, for example, by Gärdenfors (1988) - as the elaboration leaves little room for varying the three central operations (expansion, contraction, and revision) and requires severe idealization, it seems not to agree with a content-guided understanding of scientific change. Set theoretic tools were heavily used by the structuralists (Balzer, Moulines \& Sneed 1987) and probabilistic tools (Markov chains) in Pearl's (2000) theory of causality.

The examples from the previous paragraph proceed in terms of CL and extensions thereof. Sometimes alternative logics are more suitable for clarifying certain methodological concepts. Among the popular examples, I refer especially to Van Fraassen's supervaluations and to the partial structures of da Costa and associates - see Steven French's paper in this volume.

A different use of logic is made when the aim is not to define a concept, but rather to describe, in more or less detail, the stages of a reasoning process. Remark that, for example, defining an explanation of a certain kind is a very different thing from describing the process by which this kind of explanation is obtained. A typical example is presented in Kuipers \& Wiśniewski (1994). Wiśniewski's erotetic logic is invoked to characterize the "train of thought" in searching an explanation by specification. The central tool here is erotetic implication: how questions together with declarative statements imply other questions.

The most elaborate unified approach of this kind is Hintikka's work on interrogative logic (e.g. Hintikka 1999). This logic uses a variant of Beth tableaux for book-keeping - the original device is for testing inference: the premises are written on the left side, the conclusion on the right side, and next the tableau rules are applied, which sometimes causes a subtableau being started; a tableau may close, remain open, or not stop. Hintikka interprets the tableaux in a game-theoretic way, for example as a game against nature.

Consider an application to the search of an explanation for a singular statement $E$ in terms of a theory $T$. The axioms of $T$ are introduced on the left side and $E$ on the right. An explanation of $E$ in terms of $T$ requires that $E$ is not a CL-consequence of $T$. So, in order for the tableau to close, new information needs to be introduced. This is obtained by introducing questions on the left side, which requires that their presupposition occurs on the same side. The answers that nature gives are represented by a fixed set $S$; if the answer to a question is in $S$, the answer is added on the left side. Apart from the rules of 
the game, there is also a deductive heuristics as well as an interrogative heuristics - I avoid Hintikka's "strategy" for reasons that become clear below. Where the rules determine which moves are permitted, a heuristics is directed towards applying the rules in such a way that the game is won. In our example the game is won when the tableau closes, because this means that one has obtained a set of answers (singular statements) that jointly form an explanation of $E$ in terms of $T$.

Hintikka has applied his interrogative logic to many problems from the philosophy of science, among them also induction. He sees this logic as central to the logic of inquiry and to the logic of discovery, and even as a general theory of reasoning. The advantage of the distinction between rules and strategies is that the latter allow for a context-guided understanding of inquiry. The disadvantage, however, is that it is difficult to say much about heuristics in the present framework, as is apparent from Hintikka's work on the topic. Moreover, there are clearly two kinds of considerations that determine a heuristics. One of them is determined by the logical structure of the problem one tries to solve, which is here represented by the tableau one tries to close. A very different kind of considerations, however, is determined by the historical situation: that the problem one tries to solve is similar to problems solved in the past and that we know which set of moves was successful in solving the latter. The first kind of considerations can clearly be described in a systematic way - they are a matter of logic. Considerations of the second kind depend on the historical situation. On the basis of historical case studies, one may try to spell out the parameters of possible problem-solving situations as well as their possible values. Any such general theory, however, is bound to be provisional because it depicts at best the present and past situations.

\section{Logics for Methodological Concepts}

Methodological concepts give rise to forms of reasoning that are not deductive. Think about inductive generalization, abduction, interpreting an inconsistent theory as consistently as possible, handling background generalizations in the presence of exceptions, invoking theories or hypotheses that are ordered by priorities, etc. - more examples are discussed in Batens (2004). Clearly such reasoning forms are not guided by deductive logic alone. This is why one needs the proposals presented in the previous section. One may try to approach the reasoning in terms of a definition, which settles whether the result of the reasoning is an object of the suitable kind. Alternatively one may try to say more about the reasoning itself, by characterizing the "train of thought" that underlies it, or by setting it up as a specific application of interrogative logic.

Different and more radical approaches attempt a characterization by a logic. Indeed, the reasoning forms are in a clear sense logics: they assign a set of correct consequences to every set of premises. In some respects they differ from usual logics. Let us at once consider the most striking feature. Most of those forms of reasoning are dynamic in that statements that are seen as consequences at some point in the reasoning are rejected at a later point, when the reasoning has led to a better understanding of the premises. At a still later point they may be reinstated as consequences in view of the continuation of the reasoning.

The dynamics is related to the fact that many of those reasoning forms are 
non-monotonic: what follows from part of the premises need not follow from all of them. Inductive generalization, which obviously relies on background knowledge, is non-monotonic because the derived generalizations need be compatible with the data. As new data are taken into consideration, formerly derived conclusions may have to be withdrawn. The opposite move may also be justified. The data may prevent us from accepting either that all $P$ are $Q$ or that all $R$ are not- $Q$ (because, although some $P$ are known to be $Q$ and some $R$ are known to be not- $Q$, some $P$ are known to be $R$ while their $Q$-hood is unknown). If the further data reveal that some $R$ is $Q$, and hence falsify that all $R$ are not- $Q$, this may (in some circumstances) make it sound to conclude that all $P$ are $Q$.

Even monotonic reasoning processes may display the dynamics described in the next to last paragraph. Indeed, the cause of the dynamics is not nonmonotonicity, but the absence of a 'positive test' for the consequence relation (sometimes described as the fact that the consequence relation is not even partially recursive). This requires a brief digression. CL is undecidable: no mechanical procedure tells us (after finitely many steps) whether, for an arbitrary set of premises $\Gamma$ and an arbitrary statement $A, A$ is a CL-consequence of $\Gamma$ or not. However, there is a positive test for derivability: there is a mechanical procedure that, if $A$ is a CL-consequence of $\Gamma$, will inform us so after finitely many steps. For the aforementioned reasoning processes there is not even a positive test. So no mechanical procedure will, for an arbitrary $\Gamma$ and an arbitrary $A$ that is a consequence of $\Gamma$, tell us after finitely many steps that $A$ is a consequence of $\Gamma$. The absence of a positive test may be a serious handicap from a computational point of view, but is very familiar to philosophers of science. Much sound reasoning is not 'conclusive', is revisable in view of further consideration.

Approaching methodological concepts by means of logics has some advantages, which will become clear later. This is why I continue discussing the matter. I shall do so in terms of the approach I am most familiar with, the adaptive logics approach. Let us first look at the logics themselves (this section) and next consider their application in a problem-solving context (next section). My description will be informal and slightly inaccurate at some points - an accessible and up-to-date description is available in Batens (2006, §2-5) and soon in Batens (200x).

An adaptive logic (in standard format) is characterized by a triple: a lower limit logic, a set of abnormalities and a strategy. The lower limit logic is a logic of the usual type (reflexive, transitive, monotonic, and compact) that has a characteristic semantics. The set of abnormalities is a set of formulas characterized by a logical form. Abnormalities are taken to be false, until and unless the premises prevent this. Strategies need not worry us here: they are a technical device to handle cases were the premises require that at least one out of a finite set of abnormalities is true, but fail to specify which one.

Let us consider two examples that will clarify the mechanism. The first concerns inconsistencies, which is interesting because it at once clarifies the use and limits of paraconsistent logics. Readers frightened by this can move on to the second example, which extends CL with inductive generalization.

Suppose a theory $T$ was intended as consistent, and hence was formulated with CL as its underlying logic, but turns out to be inconsistent. In order to reason sensibly from $T$, one needs to replace CL by a paraconsistent logic. In doing so, however, many CL-correct inference forms become invalid. Thus 
nearly all paraconsistent logics invalidate Disjunctive Syllogism (to derive $B$ from $A$-or- $B$ and not- $A$ ). So, if one replaces CL by a paraconsistent logic PL, all conclusions that follow from the theory by Disjunctive Syllogism are lost. This leaves us with an unrealistically poor theory. Indeed, if both $A$ and not- $A$ are derivable, then $A$-or- $B$ is derivable for every $B$. So we clearly do not want Disjunctive Syllogism to apply to $A$ and $A$-or- $B$. But if both not- $C$ and $C$-or$D$ are PL-derivable from the premises, and not- $C$ is not PL-derivable from the premises, then we would want Disjunctive Syllogism to apply and hence $D$ to be a consequence. In other words, if a theory that was intended as consistent turns out to be inconsistent, we still want to interpret it as consistently as possible in order to retain whatever can be retained from the theory as originally intended. This is precisely what inconsistency-adaptive logics do. They interpret theories as consistently as possible. So an inconsistency-adaptive logic AL is characterized as expected: the lower limit logic is a paraconsistent logic and the set of abnormalities is the (existential closure) of formulas of the form $A$-and-not- $A$. By taking these as false in as far as the premises permit, the adaptive logic AL interprets the premises as consistently as possible: the AL-consequences of the premises contain all desired CL-consequences and do not contain the undesired ones (viz. do not contain all statements).

Take inductive generalization as a second example: which statements of the form "All $A$ are $B$ " can be jointly upheld in view of a given set of empirical data (which need not be primitive formulas). Realistic applications require that one takes background theories into account. Moreover, some background theories are rejected when falsified by the data, whereas others are retained except for the falsified generalizations or even except for the falsified instances of generalizations. This is realized by combining a diversity of adaptive logics for handling background generalizations with the adaptive logic for inductive generalization - space limitations force me to restrict the discussion to the latter. The lower limit logic is CL. The set of abnormalities is the set of formulas of the form something-is- $A$-and-something-is-not- $A$. This is obviously inspired by Carnap's idea of uniformity. Inductive generalization (which, incidentally, Carnap was unable to obtain in terms of his probabilistic approach) is made possible by interpreting the world as uniformly as the data permit. So abnormalities are taken to be false until and unless the data force us to consider them as true.

Adaptive logics of inductive generalization assign to every set of data, phrased in a given language, a unique set of inductive generalizations that are jointly consistent with the data - they do the same when the data are first extended in terms of background theories. This set is arguably the best set of generalizations to act upon. Moreover, Reichenbach's 'pragmatic justification of induction' applies: if a set of generalizations holds in the world, then the consequence set of the logic of inductive generalization converges towards it. There is more. The logic of inductive generalization (as other adaptive logics) may be applied to guide research, as we shall see in the next section.

There is obviously a large set of inconsistency-adaptive logics. They are obtained by varying (mainly) the paraconsistent lower limit logic. So the bad message is that inconsistency-adaptive logics require a justification: are they suitably applicable to the present situation. The good message is that the available multiplicity of paraconsistent logics makes it likely that the suitable inconsistency-adaptive logic for many specific contexts is readily available for the multiplicity see Béziau \& Carnielli (2006), the references therein, etc. 
The situation is different for the adaptive logic of inductive generalization: few sensible alternatives for the lower limit logic CL are at present available (and the strategy offers not much variation). This is largely compensated by the multiplicity of adaptive logics for handling background knowledge.

Many more adaptive logics have been studied, most of them relating to problems in the philosophy of science. Characterizing a methodological concept in terms of an adaptive logic (in standard format) has a number of attractive consequences. First, it provides an exact definition of the concept in terms of the lower limit logic and the set of abnormalities. Next, it defines the proof theory as well as the semantics of the logic. The semantics is essential for clarifying the underlying idea of the logic: it selects the lower limit models of the premises that verify only the abnormalities that are required to be true by the premises (the precise meaning of this depends on the strategy). Whatever is true in all those models is a consequence of the premises. The proof theory - basically three generic rules and a marking definition - is equally important: if offers an explication of the informal reasoning by which we try to find out whether the methodological concept applies. In this respect, the availability of dynamic proofs is one of the strongholds of adaptive logics. The basic idea is that statements that are only derivable by relying on the falsehood of certain abnormalities, are derived on a condition, viz. the set of those abnormalities. Next, it depends on the (disjunctions of) abnormalities that are derived at a certain stage of the proof whether a line is marked (and hence OUT) or unmarked (and hence IN).

The standard format itself takes care of the metatheory. It warrants that the proof theory and semantics are equivalent, and it warrants that a set of desirable metatheoretic properties are present (Batens 2006, Batens 200x), in other words that the logics do the required job in a decent way. So, as soon as one is able to characterize a methodological concept in terms of an adaptive logic in standard format, all the hard logician's work is provided for free. The standard format even provides one with a set of criteria for determining, for some premise sets $\Gamma$ and conclusions $A$, whether $A$ is or is not an adaptive consequence of $\Gamma$. Although no algorithm is available, the criteria may apply. Where they do not, the proof theory (together with the prospective dynamics which I describe below) explicates sensible reasoning towards establishing a conclusion.

\section{Formal Problem-Solving Processes}

If a methodological concept is characterized by a logic, much of the connected reasoning is explicated by the logic. For example, whether a statement $A$ is compatible with a theory $T$ is reduced to the problem whether $A$ is a COconsequence of $T$, where $\mathrm{CO}$ is the adaptive logic of compatibility. So part of Hintikka's heuristics is taken over by the logic, whereas the rest of Hintikka's heuristics should now be phrased as a heuristics with respect to the adaptive logic - $\mathrm{CO}$ in the example.

Part of the remaining heuristics still depends on the logical structure of the problem one tries to solve. As this is a matter of formal reasoning itself, it is sensible to attempt to push it into the proofs. There is indeed an easy way to do so, viz. in terms of a prospective dynamics. Let us consider the situation for CL, which will be most transparent for the reader. Suppose that one is trying 
to derive $A$ and that "if $B$, then $A$ " is one of the premises. Then one obviously can obtain $A$ by obtaining $B$ and next applying Modus Ponens. Instead of remembering this, or writing it down on a separate slice of paper, one writes $[B] A$ in the proof. On the one hand $[B] A$ expresses that $A$ can be obtained by obtaining $B$; on the other hand it is a bookkeeping device to remember one that one tries to obtain $B$. If $B$ can be obtained directly from one of the premises, one will introduce that premise and start analysing it. If $B$ itself cannot be obtained from the premises, it is analysed. Thus if $B$ is $C$-and- $D$, then one derives $[C, D] A$ from $[B] A$. The prospective dynamics can be usefully combined with marking definitions. Thus, if $[C, D] A$ occurs in the proof and $D$ turns out to be a dead end (not to be derivable from the premises), then it is useless to try to derive $C$ in order to obtain $A$. So $[C, D] A$ is a dead end itself. Similarly, if both $[C, D] A$ and $[C] A$ occur in the proof, then the former should be marked as redundant: $C$ is sufficient to derive $A$. The prospective dynamics may be spelled out for other logics than CL, including adaptive logics. The advantage is, as said before, that those parts of the heuristics that depend on the logical structure of the problem, can be written into the proof and can thus be made transparent.

A formal problem solving process is composed of a number of elements, among them a combination of logics, the prospective dynamics for these logics, an erotetic logic (resembling the logics of Wiśniewski (1996)), and a heuristics, which actually is a kind of procedure (a set of instructions to extend a given proof in a certain way in view of the lines of the proof). One starts from a problem (a set of questions of a certain type) together with the premises. The problem gives rise to a prospective statement which determines a target. This is usually followed by deductive steps. Where these come to an end, unsolved problems together with declarative statements may give rise to deriving further problems, which then again start the prospective machinery, etc.

The above schema may easily be extended. Let us consider one example. In line with Hintikka's work, the schema can be extended for example with a question answering device, which leads to the introduction of new premises. The interesting point is that adaptive logics may be used for guiding research, viz. for deciding which questions should be asked. Typically, new consequences may be derived if one succeeds in 'narrowing down' a derived disjunction of abnormalities to (a shorter disjunction or) a single abnormality. So this is one important source of 'derived' problems that may be built in into the procedure. At any point in time, scientists have a fairly good idea of the problems that can be solved by empirical means. Formal problem solving processes will guide one in deciding to make certain observations. If an experiment is required, a related problem-solving (sub)process will be started (to make the experiment easy to perform, plausibly conclusive, ....).

The plot behind the above should be clear by now. On the one hand one tries to fix (in the logic, the prospective dynamics, and the procedure) all aspects that can be mastered by formal means. On the other hand one tries to leave room for a content-guided heuristics wherever this is possible. I shall devote the last section to the latter. 


\section{Context-Guided Reasoning}

Remark for a start that, at any given point in time, the language of a scientific discipline has been molded by the discipline's past history. This obviously applies generally and is not typical for the proposals discussed in the previous section.

All adaptive logics have rules that are not validated by the lower limit logic, but would be valid if all abnormalities are false. Such rules are neither validated nor invalidated by an adaptive logic. The logic validates certain applications of the rule, viz. those that are permitted by the premises. Phrased more precisely, it depends on the disjunctions of abnormalities derivable from the premises by the lower limit logic, whether an application of such a rule is valid or invalid. In this sense, adaptive logics are a means to formally characterize a specific (but restricted) form of content-guidance.

We have seen that the multiplicity of adaptive logics allows one to select the variant that is suitable in a specific situation and forces one to justify the choice. The same applies to the choice of an erotetic logic and to the choice of the procedure that governs the prospective dynamics.

The above plot enables one to take background theories serious, while still allowing for several forms of defeasibility in view of the data (rejecting a theory, rejecting only some generalizations that follow from a theory, rejecting only only instances of such generalizations).

An equally fascinating aspect is that the above plot leaves ample room for the introduction of guesses, which may either be wild or rely on world-views and similar personal constraints. Which guesses are useful is determined by the derived disjunctions of abnormalities. The origin of the guesses is (and should be) extra-logical, but the logic (or combination of logics) guides the guess in handling it as defeasible.

The most important content-guided aspect lies obviously in the heuristics that is not determined by the formal problem-solving process itself. Let me just mention a few aspects. It will depend on this heuristics whether one tries to derive a conclusion along one road rather than the other. It will depend on the heuristics whether one recurs to an observational question, to an experimental question, or rather tries to obtain a theoretical derivation first. (The use of models is another alternative, which should as soon as possible be built in into the plot.) How one should proceed cannot be spelled out beforehand, but should be decided in view of the case under consideration, in view of what one has learned about 'the world' and about learning. So the basic demand on a plot for formal problem solving processes it that it leaves sufficient freedom for the heuristics. In order to do that, and to situate the heuristics, the logical framework has to be spelled out. This framework should be malleable. It should consist of a set of related slots that can be filled in agreement with the demands of the case under consideration. But even then the framework, just as much as the standard deductive logic, can at best be a provisional hypothesis based on what we have learned about problem solving. A good hypothesis is one that takes into account the insights of our days. But more days are to come. 


\section{References}

Balzer, W., Moulines, C. U. \& Sneed, J. D. (1987), An Architectonic for Science. The Structuralist Program, Reidel, Dordrecht.

Batens, D. (2002), In defence of a programme for handling inconsistencies, in Meheus (2002), pp. 129-150.

Batens, D. (2004), The need for adaptive logics in epistemology, in D. Gabbay, S. Rahman, J. Symons \& J. P. V. Bendegem, eds, 'Logic, Epistemology and the Unity of Science', Dordrecht, Kluwer, pp. 459-485.

Batens, D. (2006), A universal logic approach to adaptive logics, in J.-Y. Béziau, ed., 'Directions in Universal Logic', Birkhauser. In print.

Batens, D. (200x), Adaptive Logics and Dynamic Proofs. A Study in the Dynamics of Reasoning. Forthcoming.

Béziau, J.-Y. \& Carnielli, W. A., eds (2006), Paraconsistent Logic with no Frontiers, Studies in Logic and Practical Reasoning, North-Holland/Elsevier. In print.

Gärdenfors, P. (1988), Knowledge in Flux: Modeling the Dynamics of Epistemic States, MIT-press, Cambridge, Ma.

Hintikka, J. (1999), Inquiry as Inquiry: A Logic of Scientific Discovery, Kluwer, Dordrecht.

Kuipers, T. A. F. (2000), From Instrumentalism to Constructive Realism. On some Relations Between Confirmation, Empirical Progress, and Truth Approximation, Vol. 287 of Synthese Library, Kluwer, Dordrecht.

Kuipers, T. A. F. \& Wiśniewski, A. (1994), 'An erotetic approach to explanation by specification', Erkenntnis 40, 377-402.

Meheus, J., ed. (2002), Inconsistency in Science, Kluwer, Dordrecht.

Pearl, J. (2000), Causality. Models, Reasoning, and Inference, Cambridge University Press, Cambridge.

Shapere, D. (2004), Logic and the philosophical interpretation of science, in P. Weingartner, ed., 'Alternative Logics. Do sciences need them?', Springer, Berlin, Heidelberg, pp. 41-54.

Wiśniewski, A. (1996), 'The logic of questions as a theory of erotetic arguments', Synthese 109, 1-25. 